\title{
A VANISHING THEOREM ON MANIFOLDS OF POSITIVE SPECTRUM
}

\author{
YIU-Ming LO
}

\section{Introduction}

In recent work of Witten-Yau, Cai-Galloway, and X. Wang, they investigated the relation of the homology group $H_{n-1}(M, Z)=0$ under the assumption that $M$ is a conformally compact manifold of dimension $\geq 3$. The great interest relies on the close relation between this class of manifolds and the AdS/CFT correspondence which links the conformal field theory and supergravity together.

A manifold $M$ is conformally compact if its complete metric is of the form

$$
d s^{2}=\rho^{-2} d s_{0}^{2},
$$

where $d s_{0}^{2}$ is some background metric defined on the manifold with boundary $\widetilde{M}=M \cup \partial M$ and $\rho$ is a defining function satisfying

$$
\rho=0 \quad \text { on } \quad \partial M
$$

and

$$
\mathrm{d} \rho \neq 0 \quad \text { on } \quad \partial M .
$$

In his thesis [19] and [20], X. Wang proved the following theorem:

Theorem 1 (Wang). Let $M^{n}$ be a conformally compact manifold of dimension $n \geq 3$ with Ricci curvature bounded from below by

$$
\operatorname{Ric}_{M} \geq-(n-1) \text {. }
$$

Let $\lambda_{1}(M)$ denote the lower bound of the spectrum of the Laplacian on $M$. If

$$
\lambda_{1}(M) \geq n-2,
$$

then either

(1) $H^{1}\left(L^{2}(M)\right)=0$; or

(2) $M=\mathbf{R} \times N$ with the warped product metric $d s^{2}=d t^{2}+\cosh ^{2} t d s_{N}^{2}$, where $N$ is a compact manifold with Ricci curvature bounded from below by

$$
\operatorname{Ric}_{N} \geq-(n-2) \text {. }
$$

In particular, $M$ either has only one end or it must be a warped product given as above.

Received February 11, 2003.

Research partially supported by NSF grant \#DMS-0202508. 
Due to Mazzeo's theorem on conformally compact manifolds, we are able to identify the $L^{2}$-cohomology group $H^{1}\left(L^{2}(M)\right)$ with the relative cohomology group $H^{1}(M, \partial M)$ and hence we obtain the vanishing of $H^{1}(M, \partial M)$.

Later this type of theorems were generalized by Leung-Wan and Li-Wang, respectively in different directions. In [7], Leung and Wan extended X. Wang's arguments to harmonic maps and generalized his result to a wider class of manifolds, namely, the class of asymptotically hyperbolic conformally compact manifold of order $C^{1}$. In fact, they showed that:

Theorem 2 (Leung-Wan). Suppose that $\left(M^{n}, g\right), n \geq 3$ is an asymptotically hyperbolic conformally compact manifold of order $C^{1}$ such that Ric $_{M} \geq-(n-1) g$ and $\lambda_{1}(M) \geq n-2$. Suppose that $f: M \rightarrow N$ is a smooth harmonic map of finite total energy from $M$ into a complete non-positively curved manifold $N$. If $\lambda_{1}(M)>n-2$, then $f$ is a constant map. If $\lambda_{1}(M)=n-2$, then either $f$ is a constant map, or $M=\mathbf{R} \times \Sigma$ with the warped product metric $g=$ $d t^{2}+\cosh ^{2}(t) d s_{\Sigma}^{2}$, where $\left(\Sigma, d s_{\Sigma}^{2}\right)$ is a compact manifold with Ric $c_{\Sigma} \geq-(n-2)$.

As an application, they showed that the homotopy classes in $[(M, \partial M),(N, *)]$ are trivial, or $M$ splits as a warped product of the real line and some compact manifold.

In another direction, Li and Wang [12] generalized the theorem to a class of manifolds with positive spectrum.

Theorem 3 (Li-Wang). Let $M$ be a complete Riemannian manifold of dimension $n \geq 3$. Suppose $\lambda_{1}(M)>0$ and

$$
\operatorname{Ric}_{M} \geq-\frac{(n-1) \lambda_{1}(M)}{n-2} \text {. }
$$

Then either

(1) $M$ has only one end with infinite volume; or

(2) $M=\mathbf{R} \times N$ with the warped product metric $d s^{2}+\cosh ^{2}\left(\sqrt{\frac{\lambda_{1}(M)}{n-2}} t\right) d s_{N}^{2}$, where $N$ is a compact manifold with Ricci curvature bounded from below by

$$
\operatorname{Ric}_{N} \geq-\lambda_{1}(M)
$$

By combining the idea of Li-Wang and Leung-Wan, we are able to prove:

Theorem 4 (Main Theorem). Let $M$ be a complete Riemannian manifold of $d i$ mension $n \geq 3$ and $N$ be a manifold of non-positive sectional curvature. Suppose $\lambda_{1}(M)>0$ and

$$
\operatorname{Ric}_{M} \geq-\frac{(n-1) \lambda_{1}(M)}{n-2}
$$

Either $M$ splits as a warped product of the real line and a compact manifold, or any smooth map $h: M \rightarrow N$ which is constant outside a compact set is homotopic to a constant map. 
In particular, if $M$ has only one end, then for any compact set $K$ the homotopy class $[(M, M-K),(N$, a point $)]$ is trivial.

In fact, Li and Wang [12] showed that for a subclass of bounded harmonic functions with finite Dirichlet integral constructed in [10] there exists some $a$ such that

$$
\int_{E(R+1) \backslash E(R)}(f-a)^{2} \leq C \exp \left(-2 \sqrt{\lambda_{1}(E)} R\right) .
$$

By following their argument, we can show that the similar energy estimate holds for certain class of harmonic maps.

The main key of the proof is based on the fact that a power of the energy density of a harmonic map is in $L^{2}(M)$. Then the Bochner formula forces the energy density must be zero on a manifold of one end.

We remark that there is certain subtlety of our formation of our theorem. For a harmonic map $f$ which is homotopic to a map constant outside a compact set, apriorily, it might not be a constant map, although the unique continuation theorem implies that $f$ is constant if it is constant on some open set.

Throughout the whole paper, we denote $E(R)=E \cap B_{p}(R)$ and $\partial E(R)=$ $E \cap \partial B_{p}(R)$, where $E$ is an end of $M$. We also denote the bottom of the $L^{2}$ spectrum of the Laplacian on $E$ satisfying Dirichlet boundary conditions on $\partial E$ by $\lambda_{1}(E)$. Thus for any compactly supported smooth function $\phi$ on $E$

$$
\lambda_{1}(E) \int_{E} \phi^{2} \leq \int_{E}|\nabla \phi|^{2} .
$$

Acknowledgement. The author would like to thank his advisor Prof. Peter Li for his support and patience and Jiaping Wang for reading the earlier version of the paper and sharing his idea and suggestion for the improvement of the paper.

\section{Vanishing Theorem for Harmonic Maps}

First let us recall a fundamental theorem on the existence of harmonic maps with finite total energy and then we will construct a class of harmonic maps.

For a smooth map $h: M \rightarrow N$, the energy density of $h$ is defined to be

$$
e(h):=\operatorname{tr}_{M}\left(h^{*} d s_{N}^{2}\right),
$$

where $\operatorname{tr}_{M}$ is the trace with respect to the metric $d s_{M}^{2}$. The total energy of $h$ is

$$
E(h):=\int_{M} e(h) d v_{M}
$$

Theorem 5 (Schoen-Yau). Let $M$ be a complete Riemannian manifold with

$$
\operatorname{Ric}_{M} \geq-(n-1) k^{2}
$$

and $N$ be a complete manifold of non-positive sectional curvature. Let $h: M \rightarrow$ $N$ be a smooth map of finite total energy. Then there exists a harmonic map $f: M \rightarrow N$ such that $f$ is homotopic to $h$ on compact sets of $M$ and $f$ has finite energy. 
Let us give an outline of the proof for our purpose. Let $h$ be a map from $M$ to $N$ which is constant outside a compact set of $M$. Let $\Omega_{i}$ be a sequence of compact manifolds with boundary such that $M=\cup_{i} \Omega_{i}$. Then by Hamilton's theorem of Dirichlet problem for harmonic maps, we can find harmonic maps $f_{i}: \Omega_{i} \rightarrow N$ which are homotopic to $\left.h\right|_{\Omega_{i}}$. We also have

$$
E\left(f_{i}\right) \leq E\left(\left.h\right|_{\Omega_{i}}\right) .
$$

Then it is standard to show that there exists a subsequence of $f_{i}$ which converges uniformly on compact sets in $M$.

We denote by $\mathcal{K}$ the class of harmonic maps which can be constructed as above for some $h: M \rightarrow N$ which is constant outside a compact set of $M$.

Since the usual distance function is not globally smooth, we have to go through the universal coverings of $M$ and $N$ and define the homotopic distance function. For the sake of completeness, we reproduce the construction here. Let $f$ and $g$ be homotopic maps from $M$ to $N$ and let $\widetilde{M}$ and $\widetilde{N}$ be the universal covers of $M$ and $N$ respectively. Then $\pi_{1}(M, *)$ and $\pi_{1}(N, *)$ act as groups of isometries on $\widetilde{M}$ and $\widetilde{N}$ respectively so that $M=\widetilde{M} / \pi_{1}(M, *)$ and $N=\widetilde{N} / \pi_{1}(N, *)$. Let $\widetilde{r}$ be the distance function on $\widetilde{N}$. Since $\widetilde{N}$ has non-positive curvature, $\widetilde{r}$ is smooth on $\widetilde{N} \times \widetilde{N} \backslash$ diagonal. Now $\pi_{1}(N, *)$ acts on $\widetilde{N} \times \widetilde{N}$ as a group of isometries by

$$
\alpha(x, y)=(\alpha(x), \alpha(y)) \text { for } \alpha \in \pi_{1}(\mathrm{~N}, *) .
$$

Thus $\widetilde{r}$ induces a function $r: \widetilde{N} \times \tilde{N} / \pi_{1}(N, *) \rightarrow \mathbf{R}$. Let $F: M \times[0,1] \rightarrow N$ be a homotopy of $f$ with $g$ so that $F(p, 0)=f(p)$ and $F(p, 1)=g(p)$ for all $p \in M$. We now choose a lifting $\widetilde{F}: \widetilde{M} \times[0,1] \rightarrow \widetilde{N}$, and let

$$
\widetilde{f}(p)=F(p, 0) \text { and } \widetilde{g}(p)=F(p, 1)
$$

for all $p \in M$. This defines lifting $\tilde{f}, \widetilde{g}$ of $f, g$. Thus if $\gamma \in \pi_{1}(M, *)$, there exists $\alpha \in \pi_{1}(N, *)$ with

$$
\widetilde{f}(\gamma(p))=\alpha \widetilde{f}(p) \text { and } \widetilde{g}(\gamma(p))=\alpha \widetilde{g}(p) \text { for all } p \in \widetilde{M} \text {. }
$$

We define a map $\widetilde{h}: \widetilde{M} \rightarrow \widetilde{N} \times \widetilde{N}$ by $\widetilde{h}(p)=(\widetilde{f}(p), \widetilde{g}(p))$ and it induces a map

$$
h: M \rightarrow \widetilde{N} \times \widetilde{N} / \pi_{1}(N, *) .
$$

We now define $\rho(f, g): M \rightarrow \mathbf{R}$ by

$$
\rho(f, g)=r^{2} \circ h .
$$

Then $\rho(f, g)$ is smooth on $M$. We call $\rho(f, g)$ to be the homotopic distance between $f$ and $g$. Moreover, if $f$ is harmonic and $g$ is constant, then the hessian comparison theorem implies $\rho(f, g)$ is subharmonic and

$$
\Delta \rho(f, g) \geq 2 e(f) \text {. }
$$


Lemma 2.1. Let $M$ be a complete Riemannian manifold. Suppose $E$ is an end of $M$ such that $\lambda_{1}(E)>0$. Then for any smooth harmonic map $f \in \mathcal{K}$, we have the energy decay estimate

$$
\int_{E(R+1) \backslash E(R)} e(f) \leq C \exp \left(-2 \sqrt{\lambda_{1}(E)} R\right)
$$

for some constant $C>0$ depending on $f, \lambda_{1}(E)$ and $n$.

Proof of Lemma 2.1. Let $f \in \mathcal{K}$. Since the initial map of $f$ is constant outside a compact set, without loss of generality, we may assume it is constant on the end $E$. We will denote the homotopic distance between $f$ and its initial map by $\rho(f)$.

It now follows from $(2.2)$ that $\rho(f)$ is a subharmonic function on $E$ and

$$
\Delta \rho \geq 2 e(f) \geq 0 \text {. }
$$

Thus letting $\phi$ be a non-negative cut-off function on $M$ and multiplying (2.3) by $\phi^{2}$ and applying integration by parts gives

$$
\begin{aligned}
\int_{M} \phi^{2} e(f) & \leq \frac{1}{2} \int_{M} \phi^{2} \Delta(\rho(f)) \\
& =-\frac{1}{2} \int_{M}\left\langle\nabla \phi^{2}, \nabla(\rho(f))\right\rangle \\
& \leq \int_{M} \phi|\nabla \phi||\nabla \rho| \sqrt{e(f)} \\
& \leq \frac{1}{2} \int_{M} \phi^{2} e(f)+\frac{1}{2} \int_{M}|\nabla \phi|^{2}|\nabla \rho|^{2} .
\end{aligned}
$$

By using $\mid \nabla$ dist $\left.\right|^{2}=1$, we obtain

$$
\int_{M} \phi^{2} e(f) \leq C \int_{M}|\nabla \phi|^{2} \rho(f)
$$

for some absolute constant $C>0$. So it suffices to establish the decay estimate for $\rho(f)$.

Let $f_{i}$ be a sequence of harmonic maps which converge uniformly to $f$ on compact sets and $\rho_{i}: M \rightarrow \mathbf{R}$ be the corresponding homotopic distance between $f_{i}$ and the initial map. Here we extend $f_{i}$ to a constant map outside $\Omega_{i}$. Thus each $\rho_{i}$ converges uniformly to $\rho(f)$ on compact sets and satisfies

$$
\Delta \rho_{i} \geq 2 e\left(f_{i}\right) \geq 0 \quad \text { on } \quad \Omega_{i}, \quad \rho_{i}=0 \quad \text { on } \quad \partial \Omega_{i} .
$$

By scaling the metric, we may assume the $\lambda_{1}(E)=1$ and we want to prove

$$
\int_{E(R+1) \backslash E(R)} \rho(f) \leq C \exp (-2 R) .
$$

First, we show that for any $0<\delta<1$,

$$
\int_{E} \exp (2 \delta r) \rho(f) \leq \frac{C}{(1-\delta)^{2}}
$$


where $r(x)$ is the geodesic distance to the fixed point $p$ in $M$. In particular, $\rho(f)$ is in $L^{2}(E)$.

To do this, let $\phi$ be the non-negative cut-off function

$$
\phi(x)=\left\{\begin{array}{lll}
\frac{r(x)-R_{0}}{R_{0}} & \text { on } & E\left(2 R_{0}\right) \backslash E\left(R_{0}\right), \\
1 & \text { on } & E \backslash E\left(2 R_{0}\right),
\end{array}\right.
$$

and $R_{i}$ a sequence divergent to infinity. By using integration by parts and (2.5) and Cauchy-Schwarz inequality, we have for any $\epsilon>0$,

$$
\begin{aligned}
\int_{E\left(R_{i}\right)} \mid \nabla & \left.\left(\phi \exp (\delta r) \sqrt{\rho}_{i}\right)\right|^{2} \\
= & \int_{E\left(R_{i}\right)}|\nabla(\phi \exp (\delta r))|^{2} \rho_{i}+2 \int_{E\left(R_{i}\right)} \phi \exp (\delta r) \sqrt{\rho}_{i}\left\langle\nabla(\phi \exp (\delta r)), \nabla \sqrt{\rho_{i}}\right\rangle \\
& \quad+\int_{E\left(R_{i}\right)}\left(\phi \exp (\delta r)^{2}\left|\nabla \sqrt{\rho}_{i}\right|^{2}\right. \\
= & \int_{E\left(R_{i}\right)}|\nabla(\phi \exp (\delta r))|^{2} \rho_{i}-\frac{1}{2} \int_{E\left(R_{i}\right)} \phi^{2} \exp (2 \delta r) \Delta \rho_{i} \\
\quad+\int_{E(R)} \phi^{2} \exp (2 \delta r)\left|\nabla \sqrt{\rho}_{i}\right|^{2} & |\nabla(\phi \exp (\delta r))|^{2} \rho_{i}-\int_{E\left(R_{i}\right)} \phi^{2} \exp (2 \delta r) e\left(f_{i}\right) \\
\leq & \int_{E\left(R_{i}\right)}+\int_{E(R)} \phi^{2} \exp (2 \delta r) e\left(f_{i}\right) \\
= & \int_{E\left(R_{i}\right)}|\nabla(\phi \exp (\delta r))|^{2} \rho_{i} \\
\leq & (1+\epsilon) \delta^{2} \int_{E\left(R_{i}\right)} \phi^{2} \exp (2 \delta r) \rho_{i}+\left(1+\frac{1}{\epsilon}\right) \frac{1}{R_{0}} \int_{E\left(2 R_{0}\right) \backslash E\left(R_{0}\right)} \exp (2 \delta r) \rho_{i} .
\end{aligned}
$$

By using the fact that $\lambda_{1}(E)=1$ and choosing $\epsilon=\frac{1-\delta}{\delta}$, we obtain

$$
(1-\delta)^{2} \int_{E\left(R_{i}\right)} \exp (2 \delta r) \rho_{i} \leq \frac{1}{R_{0}^{2}} \int_{E\left(2 R_{0}\right) \backslash E\left(R_{0}\right)} \exp (2 \delta r) \rho_{i} .
$$

Since $\rho_{i}$ converges to $\rho(f)$ compactly uniformly, by letting $i \rightarrow \infty$, we obtain

$$
(1-\delta)^{2} \int_{E} \exp (2 \delta r) \rho(f) \leq \frac{1}{R_{0}^{2}} \int_{E\left(2 R_{0}\right) \backslash E\left(R_{0}\right)} \exp (2 \delta r) \rho(f) .
$$

Thus we have for some $C>0$

$$
\int_{E} \exp (2 \delta r) \rho(f) \leq \frac{C}{(1-\delta)^{2}} .
$$


Once we get this estimate (2.7), we can improve the estimate as in [12]. First, setting $\delta=1$ in $(2.6)$ and $\lambda_{1}(E)=1$ implies

$$
\begin{aligned}
\int_{E} \psi^{2} \exp (2 r) \rho_{i} \leq & \int_{E}|\nabla(\psi \exp (r))|^{2} \rho_{i} \\
\leq & \int_{E}|\nabla \psi|^{2} \exp (2 r) \rho_{i}+2 \int_{E} \psi \exp (2 r)\langle\nabla \psi, \nabla r\rangle \rho_{i} \\
& \quad+\int_{E} \psi^{2} \exp (2 r) \rho_{i},
\end{aligned}
$$

which gives

$$
-2 \int_{E} \psi \exp (2 r)\langle\nabla \psi, \nabla r\rangle \rho_{i} \leq \int_{E}|\nabla \psi|^{2} \exp (2 r) \rho_{i} .
$$

Since $\psi$ is a compactly supported function, by letting $i$ go to infinity, we obtain

$$
-2 \int_{E} \psi \exp (2 r)\langle\nabla \psi, \nabla r\rangle \rho \leq \int_{E}|\nabla \psi|^{2} \exp (2 r) \rho .
$$

Then we choose our cut-off function $\psi$. For $R_{0}<R_{1}<R$, let $\psi$ be

$$
\psi(x)=\left\{\begin{array}{lll}
\frac{r(x)-R_{0}}{R_{1}-R_{0}} & \text { on } & E\left(R_{1}\right) \backslash E\left(R_{0}\right) \\
\frac{R-r(x)}{R-R_{1}} & \text { on } & E(R) \backslash E\left(R_{1}\right)
\end{array}\right.
$$

Then we obtain, for any fixed $0<t<R-R_{1}$,

$$
\begin{gathered}
\frac{2 t}{\left(R-R_{1}\right)^{2}} \int_{E(R-t) \backslash E\left(R_{1}\right)} \exp (2 r) \rho(f) \\
\leq\left(\frac{2}{R_{1}-R_{0}}+\frac{1}{\left(R_{1}-R_{0}\right)^{2}}\right) \int_{E\left(R_{1}\right) \backslash E\left(R_{0}\right)} \exp (2 r) \rho(f) \\
\quad+\frac{1}{\left(R-R_{1}\right)^{2}} \int_{E(R) \backslash E\left(R_{1}\right)} \exp (2 r) \rho(f) .
\end{gathered}
$$

Based on (2.8), after an iterative argument (see [12] for details), we can show that for any positive integer $k$ and $R \geq 1$

$$
\int_{E(R) \backslash E\left(R_{0}+1\right)} \exp (2 r) \rho(f) \leq C R^{2}+2^{-k} \int_{E(R+k) \backslash E\left(R_{0}+1\right)} \exp (2 r) \rho(f)
$$

But using (2.7) and choosing $\delta$ sufficiently small, we see that the second term goes to 0 as $k \rightarrow \infty$. Thus we have

$$
\int_{E(R)} \exp (2 r) \rho(f) \leq C R^{2} \quad \text { for all } \quad R \leq R_{0} .
$$

By applying the same iterative argument, we can further improve the estimate. First, we obtain, for all $R \leq R_{0}$

$$
\int_{E(R)} \exp (2 r) \rho(f) \leq C R
$$


and repeat the iterative argument again to get

$$
\int_{E(R+2) \backslash E(R)} \exp (2 r) \rho(f) \leq C,
$$

for some constant $C>0$ independent of $R$, which implies

$$
\int_{E(R+1) \backslash E(R)} \rho(f) \leq C \exp (-2 R) .
$$

Now for $R_{0}<R_{0}+1<R<R+1$, we can choose $\phi$ in (2.4) to be

$$
\phi(x)=\left\{\begin{array}{lll}
r(x)-R_{0} & \text { on } & E\left(R_{0}+1\right) \backslash E\left(R_{0}\right) \\
1 & \text { on } & E(R) \backslash E\left(R_{0}+1\right) \\
R-r(x) & \text { on } & E(R+1) \backslash E(R) .
\end{array}\right.
$$

Then the lemma follows immediately from (2.4) and (2.10).

Now we are going to prove a vanishing theorem of harmonic maps.

Lemma 2.2. Let $M$ be a complete Riemannian manifold of dimension $n \geq 3$. Suppose $\lambda_{1}(M)>0$ and

$$
\operatorname{Ric}_{M} \geq-\frac{(n-1) \lambda_{1}(M)}{n-2} .
$$

Suppose that $f: M \rightarrow N$ is a smooth harmonic map from $M$ into a complete manifold $N$ of non-positive sectional curvature. If $f \in \mathcal{K}$ and $M$ has only one infinite volume end, $f$ must be a constant map.

Proof of Lemma 2.2. Let $f \in \mathcal{K}$ be a harmonic map constructed as above and $R$ and $K$ be the Riemannian curvature tensor of $M$ and $N$, respectively.

For a smooth harmonic map from $M$ to $N$, we have

$$
\left|\nabla^{2} f\right|^{2} \geq\left(1+\frac{1}{n-1}\right)|\nabla \sqrt{e(f)}|^{2}
$$

(See also [7], [16]). Then we apply the curvature assumption and (2.11) to the Bochner formula for harmonic maps

$$
\frac{1}{2} \Delta e(f)=\left|\nabla^{2} f\right|^{2}+R_{i j} f_{\alpha i} f_{\alpha j}-K_{\alpha \beta \gamma \delta} f_{\alpha i} f_{\beta j} f_{\gamma i} f_{\delta j}
$$

and obtain

$$
\Delta h \geq-\frac{(n-1) \lambda_{1}(M)}{(n-2)} h+\frac{|\nabla h|^{2}}{(n-1) h},
$$

where $h=\sqrt{e(f)}$. Setting $g=h^{\frac{n-2}{n-1}}=e(f)^{\frac{n-2}{2(n-1)}}$, this differential inequality can be rewritten as

$$
\Delta g \geq-\lambda_{1}(M) g
$$


Using the decay estimate and the differential inequality (2.12) and argue as in [12], we can derive an $L^{2}$ estimate of $g$

$$
\int_{B_{p}(2 R) \backslash B_{p}(R)} g^{2} \leq C R .
$$

In fact, the Cauchy-Schwarz inequality and Lemma 2.1 imply

$$
\begin{aligned}
\int_{B_{p}(2 R) \backslash B_{p}(R)} g^{2} & \\
\leq & \left(\int_{B_{p}(2 R) \backslash B_{p}(R)} \exp \left(2 \sqrt{\lambda_{1}(M)} r\right) e(f)\right)^{\frac{n-2}{n-1}} \\
& \times\left(\int_{B_{p}(2 R) \backslash B_{p}(R)} \exp \left(-2(n-2) \sqrt{\lambda_{1}(M)} r\right)\right)^{\frac{1}{n-1}} \\
\leq & C\left(\int_{B_{p}(2 R) \backslash B_{p}(R)} \exp \left(-2(n-2) \sqrt{\lambda_{1}(M)} r\right)\right)^{\frac{1}{n-1}} .
\end{aligned}
$$

Then an application of the volume comparison theorem shows that the second term in the last inequality can be bounded by $R$.

Now let $\phi$ be a non-negatively cut-off function on $M$. Since

$$
\int_{M}|\nabla(\phi g)|^{2}=\int_{M}|\nabla \phi|^{2} g^{2}+\frac{1}{2} \int_{M}\left\langle\nabla \phi^{2}, \nabla g^{2}\right\rangle+\int_{M} \phi^{2}|\nabla g|^{2},
$$

by using $\lambda_{1}(M)=1$ and integration by parts, we have

$$
\begin{aligned}
\lambda_{1}(M) \int_{M} \phi^{2} g^{2} \leq \int_{M}|\nabla(\phi g)|^{2} \\
\quad=\int_{M}|\nabla \phi|^{2} g^{2}+\frac{1}{2} \int_{M}\left\langle\nabla \phi^{2}, \nabla g^{2}\right\rangle+\int_{M} \phi^{2}|\nabla g|^{2} \\
\quad=\int_{M}|\nabla \phi|^{2} g^{2}-\frac{1}{2} \int_{M} \phi^{2} \Delta g^{2}+\int_{M} \phi^{2}|\nabla g|^{2} \\
\quad=\int_{M}|\nabla \phi|^{2} g^{2}-\int_{M} \phi^{2} g \Delta g
\end{aligned}
$$

which implies

$$
\int_{M} \phi^{2} g\left(\Delta g+\lambda_{1}(M) g\right) \leq \int_{M}|\nabla \phi|^{2} g^{2} .
$$

For $R>R_{0}$, let us choose $\phi$ such that

$$
\phi=\left\{\begin{array}{lll}
1 & \text { on } & B_{p}(R) \\
0 & \text { on } & M \backslash B_{p}(2 R)
\end{array}\right.
$$

and

$$
|\nabla \phi| \leq C / R \quad \text { on } \quad B_{p}(2 R) \backslash B_{p}(R)
$$


for some constant $C>0$. Then the right hand (2.13) can be estimated by

$$
\int_{M}|\nabla \phi|^{2} g^{2} \leq \frac{C}{R^{2}} \int_{B_{p}(2 R) \backslash B_{p}(R)} g^{2} .
$$

By the $L^{2}$ estimate of $g$, this tends to 0 as $R \rightarrow \infty$. We can conclude from (2.13) that $g$ either must be identically 0 or it must satisfy

$$
\Delta g=-\lambda_{1}(M) g \text {. }
$$

This equality forces all inequalities to be equalities. In particular, we have

$$
K_{\alpha \beta \gamma \delta} f_{\alpha i} f_{\beta j} f_{\gamma i} f_{\delta j}=0
$$

and

$$
\left|\nabla^{2} f\right|^{2}=\left.\left(1+\frac{1}{n-1}\right)|\nabla| \nabla f\right|^{2} \mid
$$

From the first equality, we conclude that $f$ must be a constant map provided that the image of $f$ has strictly negative curvature.

Otherwise, the image of $f$ is flat. Moreover, by tracing back the proof of (2.14), we have

$$
\left.|\nabla| \nabla f\right|^{2}=\left|\frac{\sum_{\alpha}\left|\nabla f^{\alpha}\right| \nabla\left|\nabla f^{\alpha}\right|}{\sqrt{\sum_{\alpha}\left|\nabla f^{\alpha}\right|^{2}}}\right|^{2}=\sum_{\alpha}|\nabla| \nabla f^{\alpha}||^{2},
$$

where $f^{\alpha}$ 's are components of the harmonic map $f$ with respect to the normal coordinates on $N$. From the equality of triangle inequality and Cauchy-Schwarz inequality, we have the vector $\nabla\left|\nabla f^{1}\right|, \ldots, \nabla\left|\nabla f^{m}\right|$ are nonnegative multiples of a nonzero one provided that they are not all zero and

$$
\left|\nabla f^{\alpha}\right|=c|\nabla| \nabla f^{\alpha}||
$$

for some $c$. We also have

$$
\left|\nabla^{2} f^{\alpha}\right|^{2}=\left.\left(1+\frac{1}{n-1}\right)|\nabla| \nabla f^{\alpha}\right|^{2} \mid \quad \text { for each } \alpha .
$$

Now, from the argument of X. Wang (see [20], [21]), we conclude that for each $\alpha, \nabla f^{\alpha}$ is a scalar multiple of $\nabla\left|\nabla f^{\alpha}\right|$. Therefore, the image under $d f$ is of rank 1 provided $\nabla f^{1}, \ldots, \nabla f^{m}$ are not all zero. It implies that the image of $f$ is contained by a geodesic in $N$.

Thus $f: M \rightarrow f(M)$ is a harmonic map of rank 1 and we have 2 cases to consider: either $f(M)$ is contained in $\mathbf{R}$ or $\mathbf{S}^{1}$. If $f(M)$ is contained in $\mathbf{R}$, then $f$ can be considered as a harmonic function. By the construction of $f$ and $\lambda_{1}(M)>0$, we have

$$
\int_{M} f^{2} \leq \lambda_{1}(M) \int_{M}|\nabla f|^{2}=\lambda_{1}(M) \int_{M} e(f)<\infty
$$

provided that $f$ is a limit of compactly supported functions. Thus $f$ is a $L^{2}$ harmonic function, and hence must be constant. Otherwise, $f$ must not be constant and hence the argument of Li-Wang implies that $M$ splits into a warped product with 2 infinite volume ends. So we have a contradiction. 
If $f(M)$ is contained by $\mathbf{S}^{1}$, then $f$ can be identified as a harmonic 1 -form on $M$ with integral period. Thus this case is reduced to the case considered by X. Wang. If $f$ is nontrivial, then X. Wang's argument again shows that $M$ splits into a warped product with 2 infinite volume ends, which contradicts to the assumption. Thus $f$ must be constant.

In view of Lemma 2.2 , we have

Theorem 2.3. Let $M$ be a complete Riemannian manifold of dimension $n \geq 3$ and $N$ be a manifold of non-positive sectional curvature. Suppose $\lambda_{1}(M)>0$ and

$$
\operatorname{Ric}_{M} \geq-\frac{(n-1) \lambda_{1}(M)}{n-2}
$$

Let $h$ be any smooth map from $M$ to $N$ which is constant outside a compact set. Then either $M$ splits into $\mathbf{R} \times N$ with the warped product metric $d s^{2}+\cosh ^{2}\left(\sqrt{\frac{\lambda_{1}(M)}{n-2} t}\right) d s_{N}^{2}$, where $N$ is a compact manifold with Ricci curvature bounded from below by $\operatorname{Ric}_{N} \geq-\lambda_{1}(M)$, or $h$ is homotopic to a constant map.

Corollary 2.4. Under the same assumption on $M$ and $N$, if $M$ has only one end, then any map $h$ constant outside a compact set is homotopic to a constant map. In particular, for any compact set $K$ the homotopy class $[(M, M-$ $K),(N$, a point)] is trivial.

\section{References}

[1] M. Cai \& G. J. Galloway, Boundaries of zero scalar curvature in the AdS/CFT correspondence, Adv. Theor. Math. Phys. 3 (1999) 1769-1783.

[2] J. Eells \& J. H. Sampson, Harmonic mappings of Riemannian manifolds, Amer. J. Math. 86 (1964), 109-160.

[3] R. S. Hamilton, Harmonic maps of manifolds with boundary, Lecture Notes in Mathematics, Vol. 471, Springer-Verlag, Berlin-New York, 1975.

[4] C. R. Graham \& J. M. Lee, Einstein metrics with prescribed conformal infinity on the ball, Adv. Math. 87 (1991), 186-225.

[5] R. E. Greene \& H. Wu, Function theory on manifolds which possess a pole, Lecture Notes in Mathematics, Vol. 699, Springer-Verlag, Berlin-New York, 1979.

[6] J. M. Lee, The spectrum of an asymptotically hyperbolic Einstein manifold, Comm. Ana. Geom. 3 (1995), 253-271.

[7] N. C. Leung \& T. Wan, Harmonic maps and the topology of conformally compact Einstein manifolds, Math. Res. Lett. 8 (2001), 801-812.

[8] P. Li, Lecture Notes on Geometric Analysis, Lecture Notes Series No. 6 - Research Institute of Mathematics and Global Analysis Research Center, Seoul National University, Seoul, 1993.

[9] P. Li \& R. Schoen, $L^{p}$ and mean value properties of subharmonic functions on Riemannian manifolds, Acta Math. 153 (1984), 279-301.

[10] P. Li \& L.-F. Tam, Positive harmonic functions on complete manifolds with nonnegative curvature outside a compact set, Ann. of Math. (2) 125 (1987), 171-207. 
[11] P. Li \& L.-F. Tam, The heat equation and harmonic maps of complete manifolds, Invent. Math., 105 (1991), 1-46.

[12] P. Li \& J. Wang, Complete manifolds with positive spectrum, J. Diff. Geom. 58 (2001), 501-534.

[13] P. Li \& J. Wang, Complete manifolds with positive spectrum, II, J. Diff. Geom. 62 (2002), 143-162.

[14] R. Mazzeo, The Hodge cohomology of a conformally compact metric, J. Diff. Geom. 28 (1988), 309-339.

[15] J. H. Sampson, Some properties and applications of harmonic mappings, Ann. Ec. Norm. Sup. 11 (1978), 211-228.

[16] R. Schoen \& S.-T. Yau, Harmonic maps and the topology of stable hypersurfaces and manifolds with non-negative Ricci curvature, Comment. Math. Helv. 51 (1976), 333-341.

[17] R. Schoen \& S.-T. Yau, Compact group actions and the topology of manifolds with nonpositive curvature, Topology 18 (1979), no. 4, 361-380.

[18] D. Sullivan, Related aspects of positivity in Riemannian geometry, J. Diff. Geom. 25 (1987), 327-351.

[19] X. Wang, On the geometry of conformally compact Einstein manifolds, PhD. thesis of Stanford University, 2001.

[20] X. Wang, On conformally compact Einstein manifolds, Math. Res. Lett. 8 (2001), 671688.

[21] X. Wang, On the $L^{2}$ cohomology of a convex cocompact hyperbolic manifold, Duke Math. J. 115 (2002) 311-327

[22] E. Witten \& S.-T. Yau Connectedness of the boundary in the AdS/CFT correspondence, Adv. Theor. Math. Phys. 3 (1999), 1635-1655.

Department of Mathematics, University of California, Irvine, CA 92697-3875

E-mail address: ylo@math.uci.edu 